Marquette University

e-Publications@Marquette

School of Dentistry Faculty Research and

Publications

Dentistry, School of

$10-1-2008$

\title{
A Technique for Duplicating a Fixed Complete Denture
}

Soni Prasad

Marquette University, soni.prasad@marquette.edu

Edward A. Monaco, Jr.

State University of New York at Buffalo

Accepted version. The Journal of Prosthetic Dentistry, Vol. 100, No. 4 (October 2008): 316-319. DOI. (C) 2008 Elsevier B.V. Used with permission.

Soni Prasad was affiliated with State University of New York at Buffalo at the time of publication. 
Marquette University

\section{e-Publications@Marquette}

\section{Dentistry Faculty Research and Publications/School of Dentistry}

This paper is NOT THE PUBLISHED VERSION; but the author's final, peer-reviewed manuscript.

The published version may be accessed by following the link in the citation below.

The Journal of Prosthetic Dentistry, Vol. 100, No. 4 (October, 2008): 316-319. DOI. This article is (C) Elsevier and permission has been granted for this version to appear in ePublications@Marquette. Elsevier does not grant permission for this article to be further copied/distributed or hosted elsewhere without the express permission from Elsevier.

\section{CONTENTS}

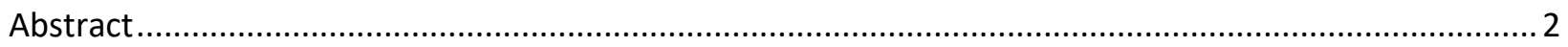

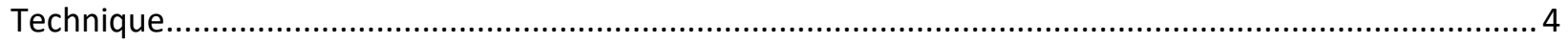

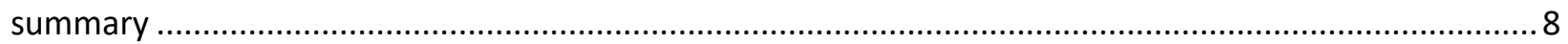

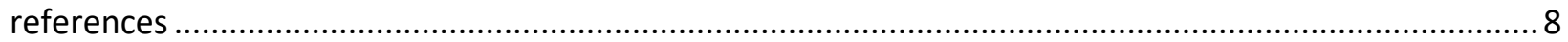

\section{A TECHNIQUE FOR DUPLICATING A FIXED COMPLETE DENTURE}

\section{Soni Prasad}

School of Dental Medicine, State University of New York at Buffalo, Buffalo, NY

Edward A. Monaco

Department of Restorative Dentistry, School of Medicine, State University of New York at Buffalo, Buffalo, NY 


\section{ABSTRACT}

The fixed implant prosthesis often requires repair due to fracture and/or wear of acrylic resin teeth. Repair of such a prosthesis, although simple, requires retaining the prosthesis to be revised in the laboratory. In order to reveneer the implant prosthesis, a provisional restoration should be fabricated for the patient that provides similar function and esthetics. This article presents a technique for duplicating the existing fixed implant-supported prosthesis prior to repair/reveneer, thus providing a smooth transition from the provisional to the definitive prosthesis.

The concept of the fixed implant prosthesis was introduced by Brånemark and initially was primarily used to restore an edentulous mandibular arch. ${ }^{1}$ The protocol involved placing 4 to 6 implants in the edentulous mandible in the interforaminal region. After an undisturbed healing period, the implants were exposed and restored with screw-retained fixed prostheses. ${ }^{1}$

Zarb et $\mathrm{al}^{2}$ and Lundqvist et $\mathrm{al}^{3}$ modified the original design of the implant prosthesis by substituting type IV gold for a $2 \%$ gold content alloy cast metal substructure and fabricating the superstructure with denture teeth and heat-polymerized acrylic resin. This design has become popular and has evolved with the use of laser-welded titanium frameworks and, more recently, computer-aided design/computeraided manufacturing (CAD/CAM) titanium frameworks. Adell et al ${ }^{4}$ reported a high long-term success rate with the combination of a gold alloy framework and a superstructure of acrylic resin. Although this technique has been used successfully for a number of years, it is not without shortcomings. ${ }^{5}$

The fixed implant-supported prosthesis often requires repair/re-veneer due to fracture and/or wear of acrylic resin teeth.6, 7 Replacing the veneer of the implant-supported prosthesis, although simple, requires retaining the prosthesis to perform the necessary laboratory procedures. To accomplish the repair/reveneer, a provisional prosthesis should be fabricated for the patient. The provisional prosthesis should provide adequate function and esthetics. Traditionally, the patient's previous dentures were relined to accommodate the healing abutments. This technique was, however, less than ideal, although it remains an option. Clearly, reintroducing a tissue-borne prosthesis after a patient has acclimated to a fixed implant-supported prosthesis requires significant accommodation from the patient during the revision period. With a modest increase in cost and a few additional patient visits, a screw-retained duplicate of the existing prosthesis can be fabricated and inserted for the interim period of revision. A technique for duplicating the patient's existing fixed implant prosthesis before it is retained for repair, thus providing a smooth transition from the provisional to the definitive prosthesis, is presented.

An 82-year-old woman presented for repair/reveneer of her mandibular fixed complete denture prosthesis, which was supported by five $3.75 \times 10-\mathrm{mm}$ implants (Brånemark; Nobel Biocare $A B$, Göteborg, Sweden). Intraoral examination of the mandibular prosthesis revealed fractured acrylic resin and worn denture teeth (Fig. 1, Fig. 2). The opposing dentition consisted of a conventional maxillary complete denture which exhibited inadequate retention and stability, as well as worn denture teeth. Both maxillary and mandibular prostheses were 11 years old. The treatment plan consisted of fabricating a new maxillary complete denture. Since the metal framework of the fixed mandibular complete denture appeared to be acceptable, the treatment plan for the mandibular arch consisted only of replacing the denture teeth and gingival resin veneer of the implant prosthesis. A provisional 
mandibular implant-supported prosthesis was planned to be fabricated and provided to the patient during the interval when the existing prosthesis was being reveneered.

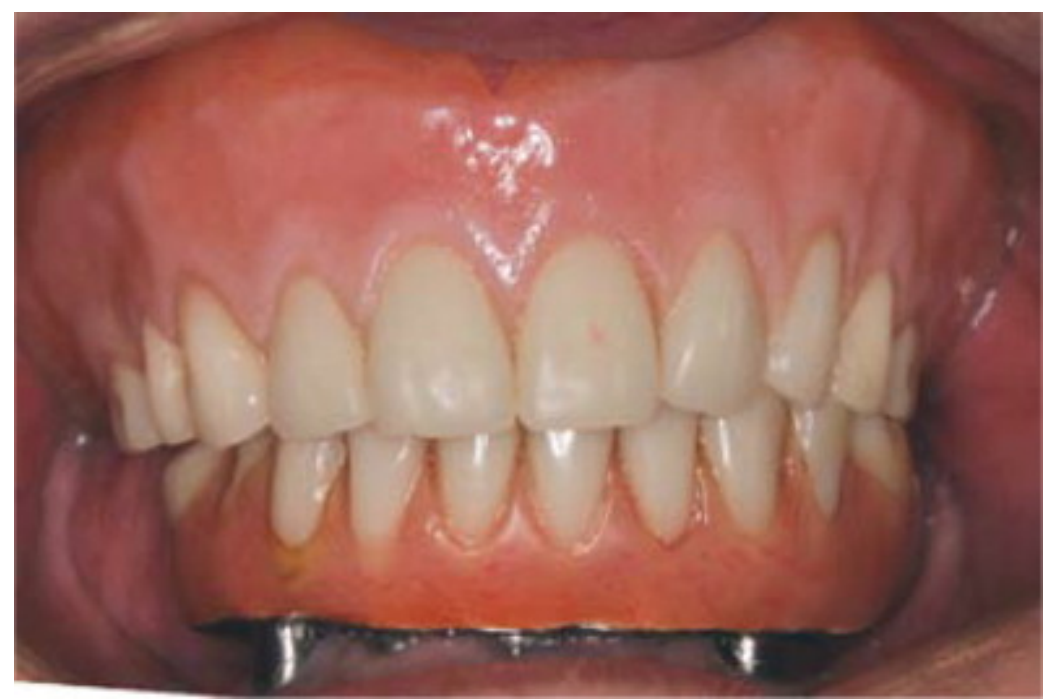

Fig. 1. Existing maxillary and mandibular prostheses in occlusion.

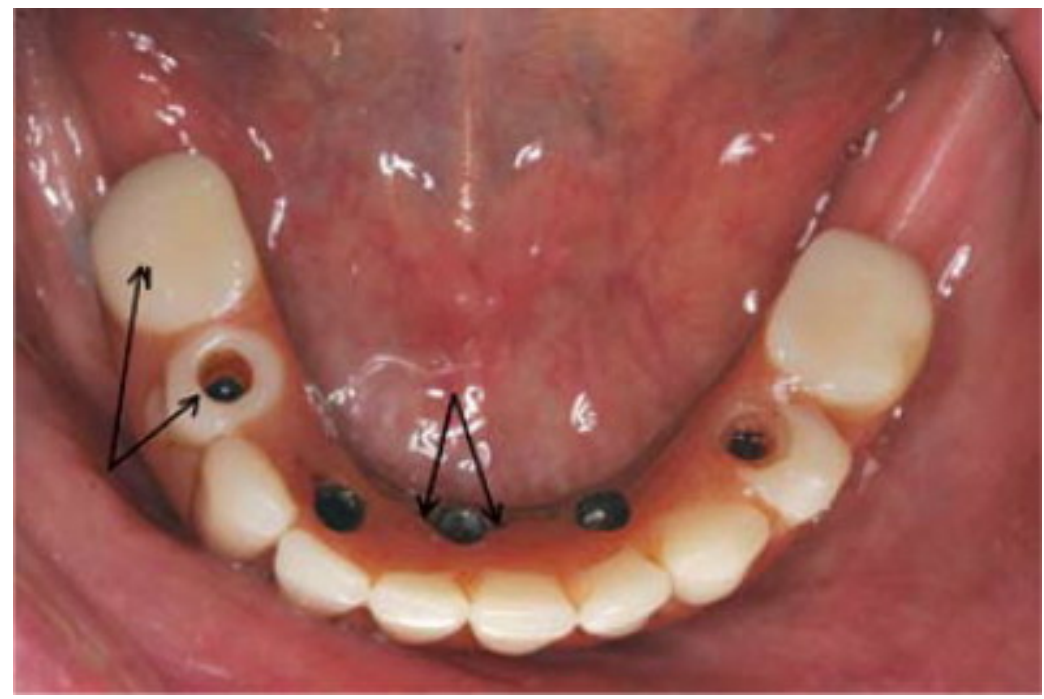

Fig. 2. Occlusal view of existing mandibular implant prosthesis with worn denture teeth and fractured acrylic resin.

The treatment phase consisted of fabricating a conventional maxillary denture and performing a maxillary complete contour wax trial arrangement by assessing phonetics, esthetics, and function. A maxillomandibular record was made with the maxillary wax trial denture and existing mandibular fixed complete denture to facilitate the mounting of the implant denture on the articulator for reveneering at a later time. The mandibular fixed complete denture was then removed for duplication. The technique for duplicating an existing fixed implant prosthesis before it is retained for repair is described. 


\section{TECHNIQUE}

1. Attach the implant analogues (Brånemark; Nobel Biocare $A B$ ) to the mandibular prosthesis (Fig. 3), and pour a base in type IV dental stone (Jade Stone; Whip Mix Corp, Louisville, Ky). Use the original definitive cast if available.

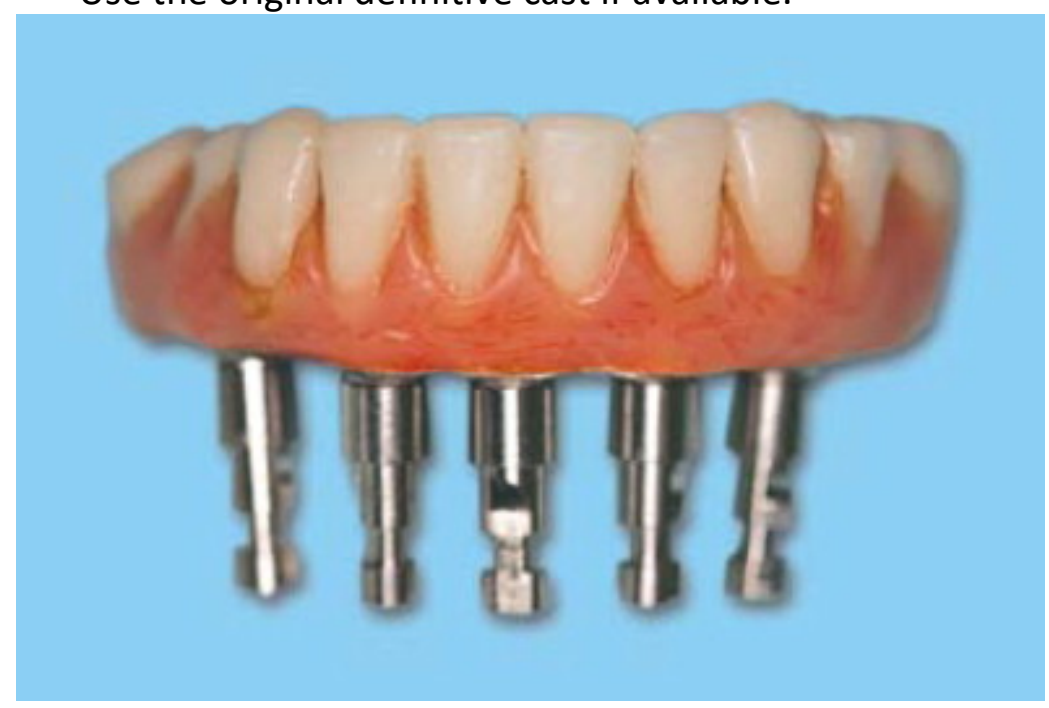

Fig. 3. Implant analogues attached to mandibular prosthesis.

2. Invest the cast in the bottom half of a flask (Hanau Varsity; Whip Mix Corp) using type III dental stone (Snap-Stone; Whip Mix Corp) (Fig. 4).

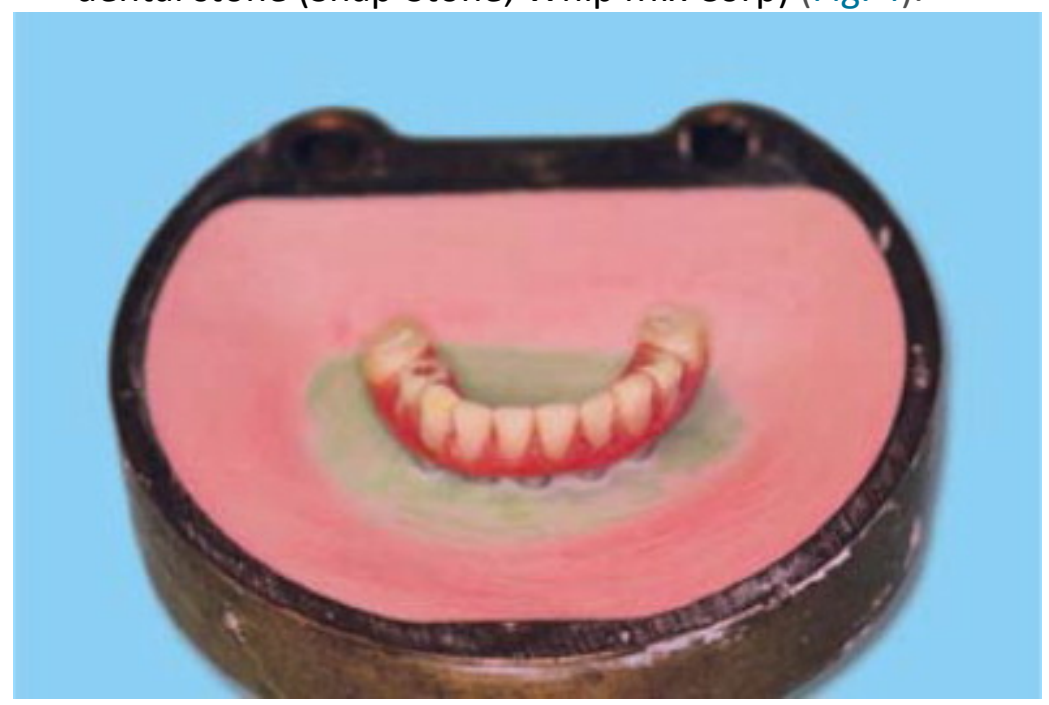

Fig. 4. Cast invested in bottom half of flask with type III dental stone.

3. Adapt silicone laboratory putty (Lab Putty; Coltène/Whaledent, Cuyahoga Falls, Ohio) in the half flask in such a manner that only the occlusal surfaces of the acrylic resin teeth are exposed (Fig. 5). 


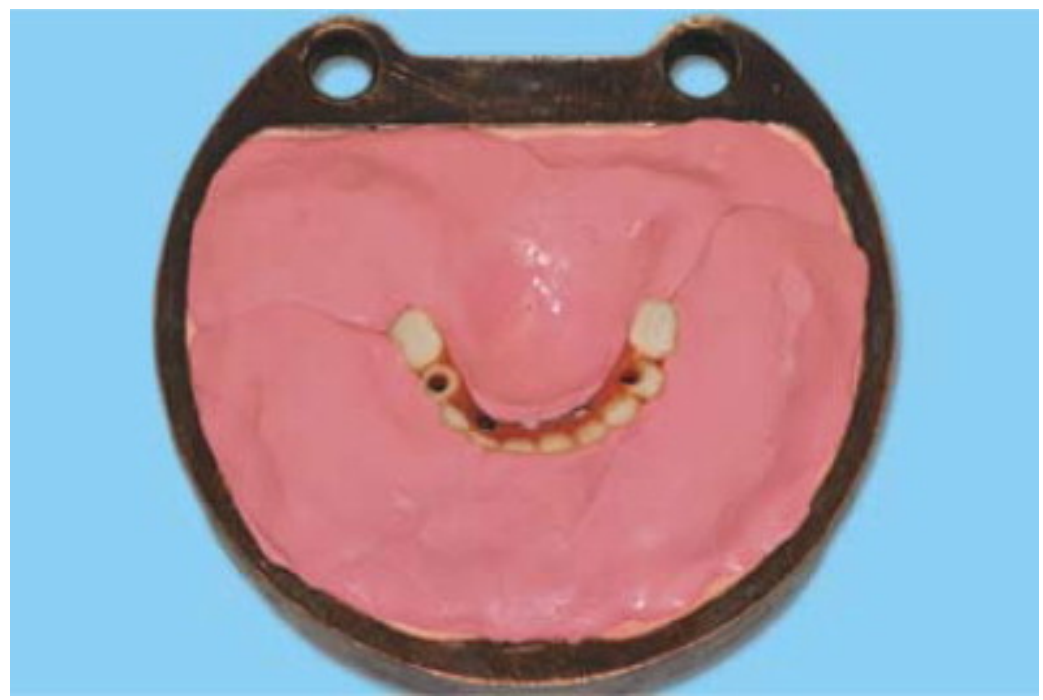

Fig. 5. Flask with first layer of laboratory putty covering prosthesis, with only occlusal surfaces of artificial teeth visible.

4. Allow the putty to polymerize and apply a silicone separating agent (Mold Release Silicone Spray; American Dental Supply, Allentown, Pa) to this layer.

5. Adapt a second layer of laboratory putty on the top of the first layer and incorporate paper pins for retention (Fig. 6).

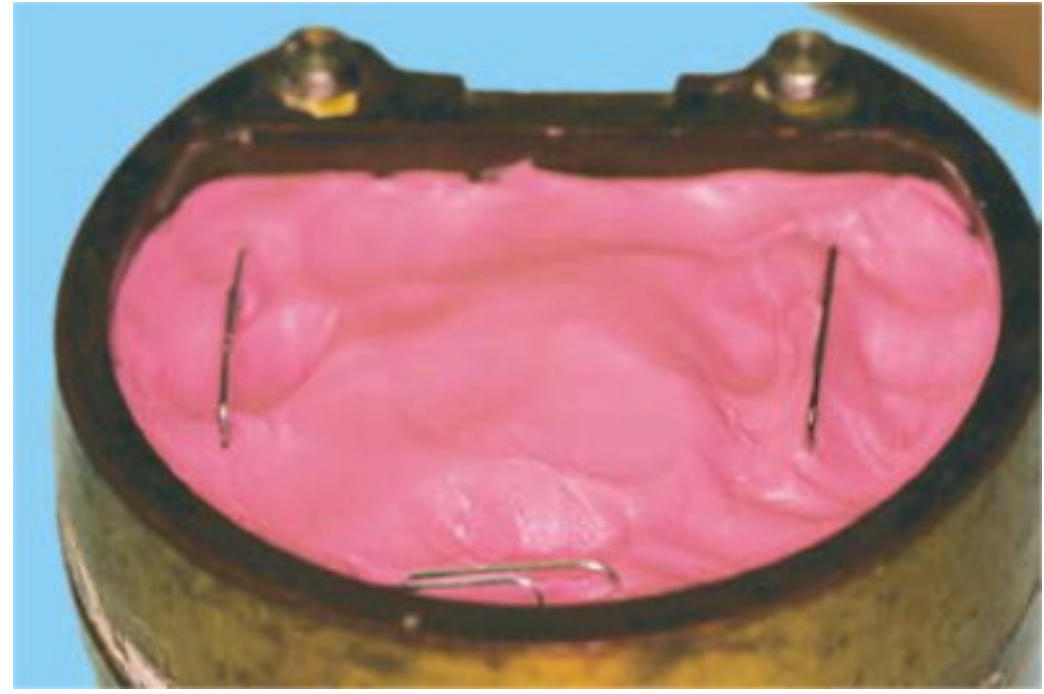

Fig. 6. Flask with second layer of laboratory putty completely covering prosthesis and incorporated pins for retention of dental stone. 
6. After the second layer of laboratory putty has polymerized, complete the flasking with type IV dental stone (Snap-Stone; Whip Mix Corp). After the stone is set, separate the flask and recover the prosthesis. Return the prosthesis to the patient.

7. Place the temporary nonengaging implant-level plastic abutments (Nobel Biocare $A B$ ) on the cast with implant analogues (Fig. 7, Fig. 8). Apply insulating putty (Dentsulate Insulating Putty; Dentsply Intl) to the abutment screws to avoid trapping acrylic resin within the abutment screw channels, to allow easy retrieval of the screws.

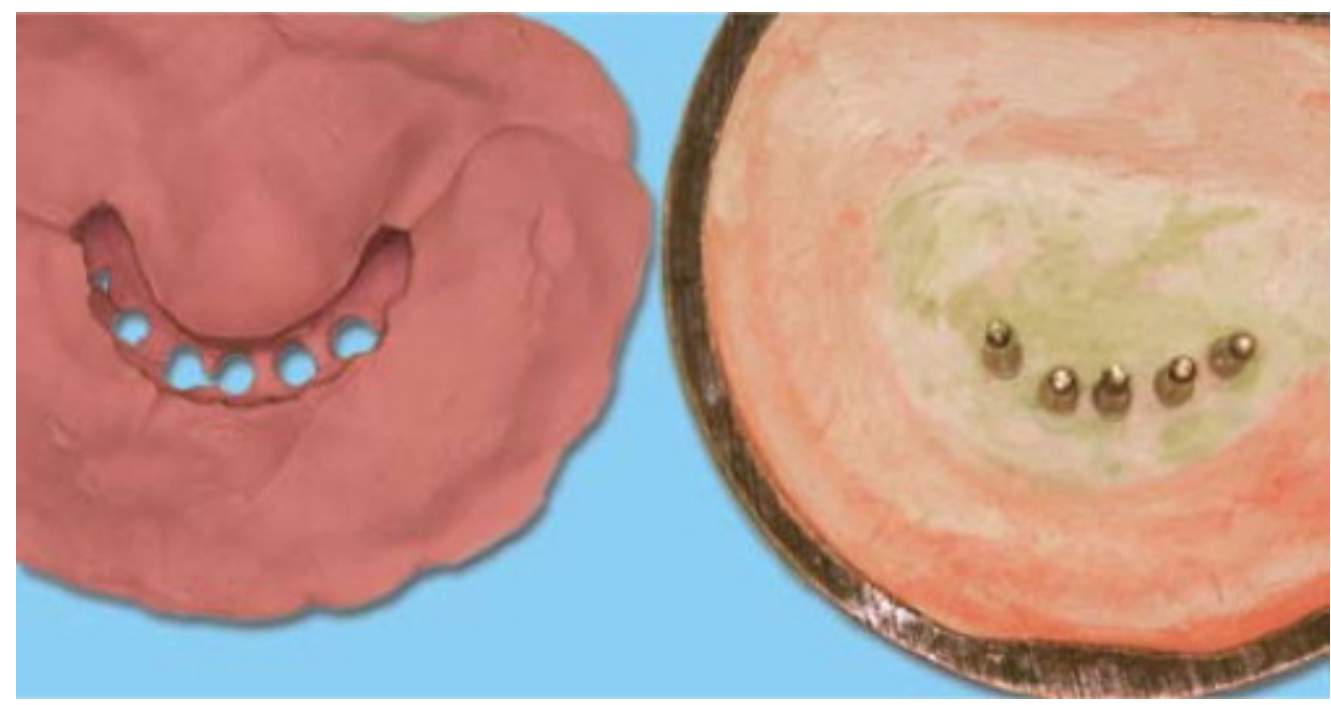

Fig. 7. Nonengaging plastic abutments on cast (right) with first layer of lab putty.

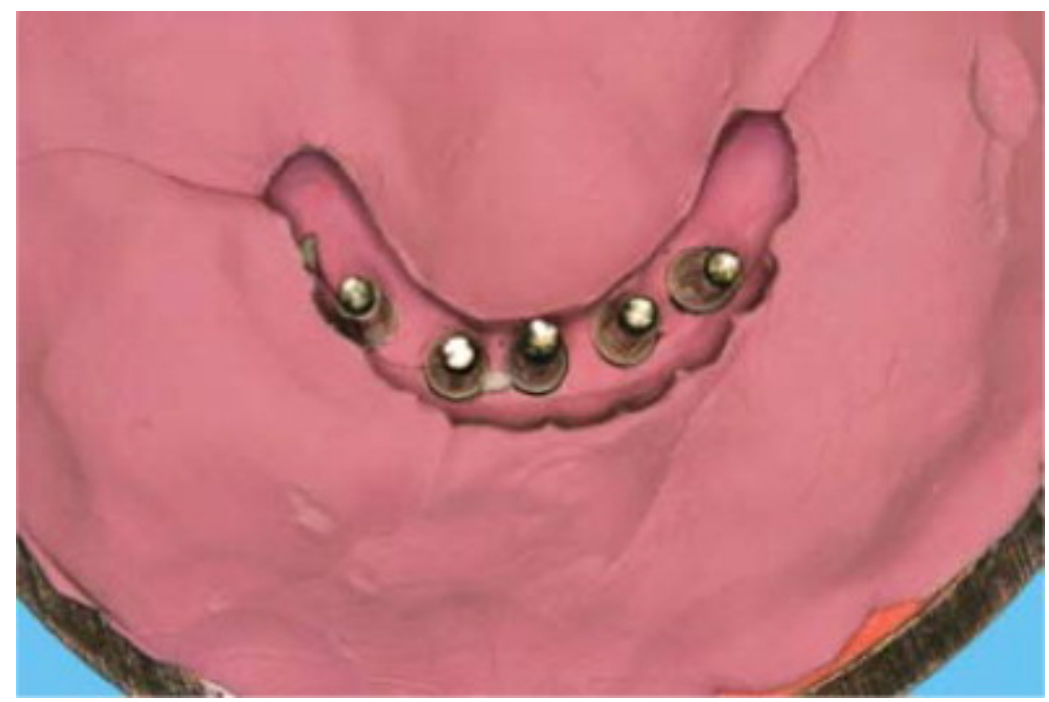

Fig. 8. Putty mold of existing prosthesis with temporary plastic cylinders in position. 
8. Pack a matching shade of heat-polymerized acrylic resin (Acralon Tooth Shade Acrylic; Henry Schein, Inc, Melville, NY) into the putty mold, close the flask, and clamp it to 1000 psi. Place the flask in a polymerizing tank at $165^{\circ} \mathrm{C}$ for 1 hour, according to the manufacturer's instructions. After the completion of polymerization, separate the flask and retrieve the provisional prosthesis. Trim away the excess acrylic resin.

9. After fabrication of the prosthesis, apply a pink acrylic resin stain (Kayon synthetic resin; Kay See Dental Mfg, Kansas City, Mo) coloring the gingival portion of the prosthesis to give it a natural appearance. Polish and finish the provisional prosthesis. Alternatively, fabricate a replica of the teeth in tooth-colored resin, and then fabricate a "framework" by linking the temporary cylinders with acrylic resin. Section this framework with a laboratory diamond disc (serrated laboratory diamond disc; Brasseler USA, Savannah, Ga) and relute the sections, one pair at a time, to minimize distortion. Reinforce the acrylic substructure using a half-round heavy gauge wire (Keystone Industries, Cherry Hill, NJ) and/or bonded reinforcement ribbon (Ribbond reinforcement ribbon; Ribbond, Seattle, Wash) prior to processing the veneering gingival shade of acrylic resin. This alternative procedure provides an advantage of increased strength and controlled polymerization shrinkage in the prosthesis which could otherwise result in undue stresses in the provisional prosthesis.

10. At this stage, remove the patient's existing prosthesis and evaluate the provisional fixed implant prosthesis intraorally. Hand tighten the abutment screws. Evaluate and adjust the occlusion intraorally (Fig. 9, Fig. 10).

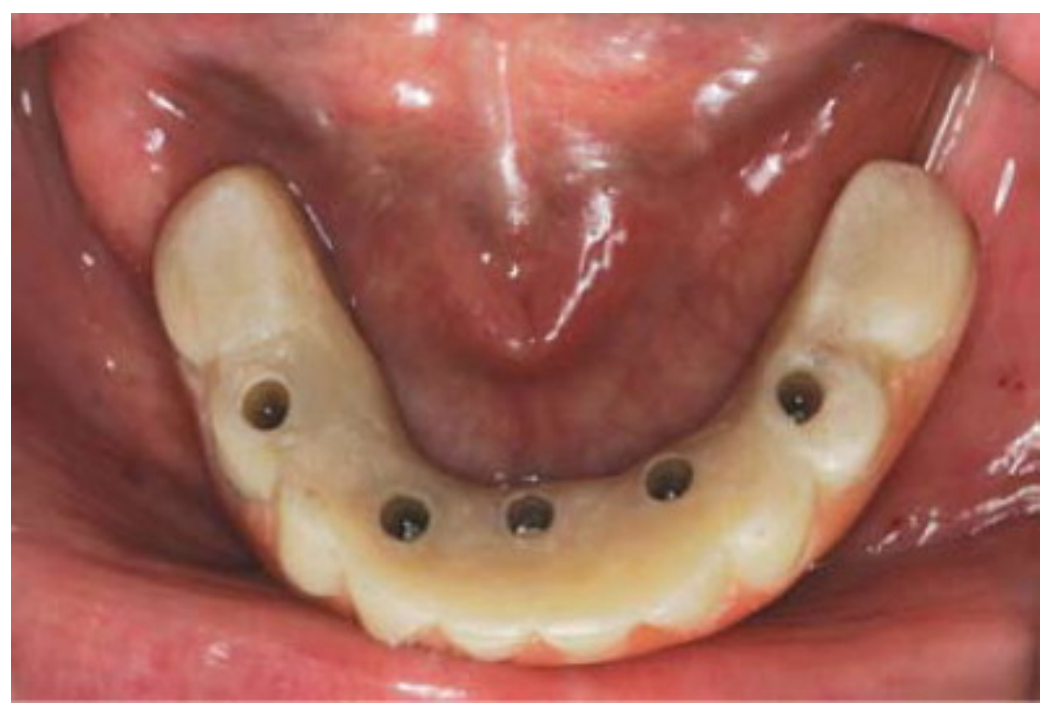

Fig. 9. Occlusal view of completed mandibular provisional implant prosthesis. 


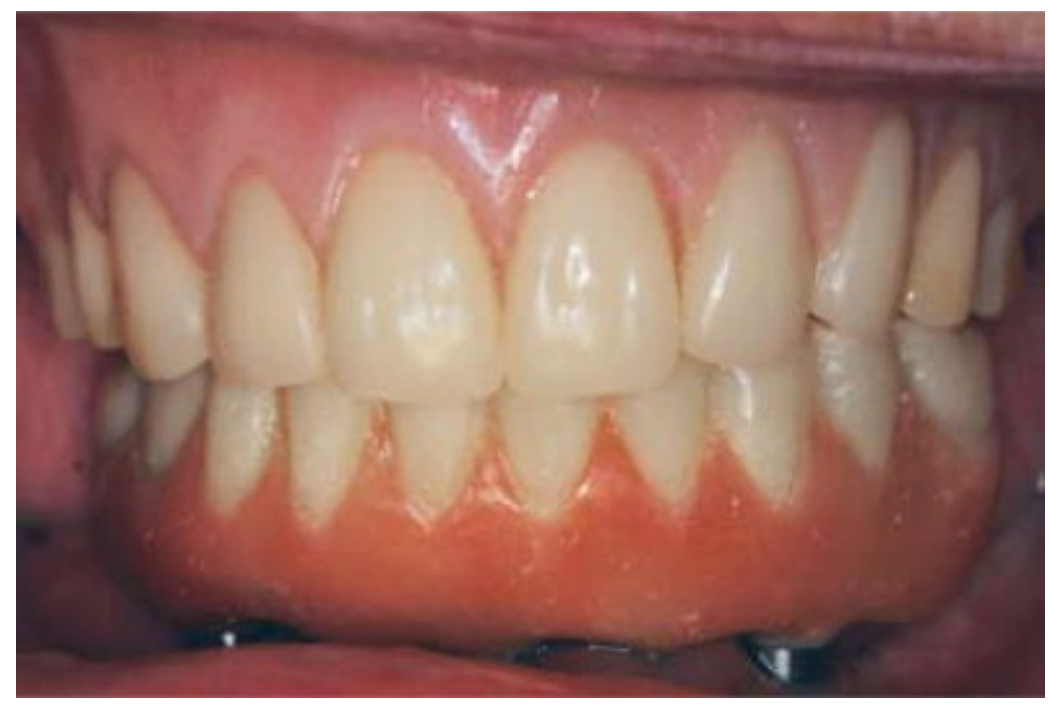

Fig. 10. Frontal view of mandibular provisional implant prosthesis in occlusion opposing existing maxillary complete denture.

11. Mount the existing implant denture using the maxillomandibular record made previously with the new maxillary wax denture and existing mandibular implant denture. Replace the denture teeth and reveneer the gingival acrylic resin of the existing mandibular implant prosthesis against the waxed new maxillary complete denture. To maintain the occlusal vertical dimension established at the time of the trial insertion of the maxillary teeth, arrange the maxillary posterior teeth superiorly to ensure adequate space for the new mandibular posterior teeth.

12. Process and insert both the maxillary complete denture and mandibular implant prosthesis simultaneously.

\section{SUMMARY}

Due to the fracture/wear of acrylic resin and/or artificial teeth, fixed complete dentures often require repair or reveneering over the course of time. With the technique described, a provisional prosthesis can be fabricated while the original prosthesis is being repaired. The provisional prosthesis fabricated in this manner is a replica of the original prosthesis, allowing similar function and esthetics and providing a smooth transition from the provisional to the definitive prosthesis.

\section{REFERENCES}

PI Brånemark, Osseointegration and its experimental background. J Prosthet Dent, 50 (1983), pp. 399-410

GA Zarb, JM Symington. Osseointegrated dental implants: preliminary report on a replication study. J Prosthet Dent, 50 (1983), pp. 271-276 
S Lundqvist, GE Carlsson. Maxillary fixed prostheses on osseointegrated dental implants. J Prosthet Dent, 50 (1983), pp. 262-270

R Adell, B Eriksson, U Lekholm, PI Brånemark, T Jemt. Long-term follow-up study of osseointegrated implants in the treatment of totally edentulous jaws. Int J Oral Maxillofac Implants, 5 (1990), pp. 347-359

GW Cobb Jr, A Metcalf, D Parsell, GW Reeves. An alternate treatment method for a fixed-detachable hybrid prosthesis: a clinical report. J Prosthet Dent, 89 (2003), pp. 239-243

$\mathrm{T}$ Jemt. Fixed implant-supported prostheses in edentulous maxilla. A five-year follow-up report. Clin Oral Implants Res, 5 (1994), pp. 142-147

GA Zarb, A Schmitt. The longitudinal clinical effectiveness of osseointegrated dental implants: the Toronto study. Part III: Problems and complications encountered. J Prosthet Dent, 64 (1990), pp. 185-194 